TITLE:

\title{
Magnetic Field Design of Coil- Dominated Magnets Wound With Coated Conductors
}

\section{$\operatorname{AUTHOR}(\mathrm{S})$ :}

Takahashi, Keita; Amemiya, Naoyuki; Nakamura, Taketsune; Mori, Yoshiharu; Ogitsu, Toru; Yoshimoto, Masahiro; Watanabe, Ikuo; Yoshiyuki, Takeshi

\section{CITATION:}

Takahashi, Keita ...[et al]. Magnetic Field Design of Coil-Dominated Magnets Wound With Coated Conductors. IEEE Transactions on Applied Superconductivity 2012, 22(3): 4901705.

\section{ISSUE DATE:}

2012-06

URL:

http://hdl.handle.net/2433/158280

\section{RIGHT:}

(C) 2012 IEEE. Personal use of this material is permitted. Permission from IEEE must be obtained for all other uses, in any current or future media, including reprinting/republishing this material for advertising or promotional purposes, creating new collective works, for resale or redistribution to servers or lists, or reuse of any copyrighted component of this work in other works.; この論文は出版社版でありません。引用の際には出版社版をご確認ご利用ください。;This is not the published version. Please cite only the published version. 


\title{
Magnetic Field Design of Coil-Dominated Magnets Wound With Coated Conductors
}

\author{
Keita Takahashi, Naoyuki Amemiya, Taketsune Nakamura, Yoshiharu Mori, Toru Ogitsu, \\ Masahiro Yoshimoto, Ikuo Watanabe, and Takeshi Yoshiyuki
}

\begin{abstract}
Coil-dominated magnets wound with coated conductors were designed for an FFAG accelerator for carbon therapy, which was designed by applying linear approximation. When designing the coil-end of the magnets, we applied differential geometry. To apply the differential geometry to three-dimensional windings with coated conductors, we introduced the concept of generalized flat-wise bending. Thereby, the combination of flat-wise bending and torsion was considered as bending of developable surface. Generated magnetic field was calculated, and the positions of conductors were optimized to minimize the field error. Three dimensional shapes of magnets were successfully designed with constraints on generalized flat-wise bending and edge-wise bending of coated conductors.
\end{abstract}

Index Terms-accelerator magnets, carbon therapy, coated conductor, high temperature superconductors

\section{INTRODUCTION}

$\mathrm{T}$ HE applications of high- $T_{\mathrm{c}}$ superconductors to accelerator magnets are attractive from the viewpoint of thermal stability and high magnetic field generation [1]-[3]. In this paper, we focus on coil-dominated magnets, which ensure the generation of precise magnetic field by designing positions of conductors precisely [4]. The objective of this paper is to show a theory for three-dimensional winding design with coated conductors, and to show the design of coil-dominated magnets for an FFAG ("Fixed-field alternating-gradient") accelerator [5] for carbon therapy. Superconducting magnets contribute to downsizing and lightening the accelerator for carbon therapy.

\section{DESIGN OF FFAG ACCELERATOR}

The FFAG accelerator uses fixed fields and allows the beam orbit to be displaced. The displacement of the beam orbit is suppressed by the magnetic field shown as

Manuscript received 8 September 2011. This work was supported by Japan Science and Technology Agency under the Strategic Promotion of Innovative Research and Development Program.

K. Takahashi, N. Amemiya, and T. Nakamura are with Kyoto University, Kyoto 615-8510, Japan (phone: +81 75383 2220; fax: +81 75383 2224; email: amemiya@kuee.kyoto-u.ac.jp).

Y. Mori is with Kyoto University, Kumatori 590-0494, Japan (email: mori@ rri.kyoto-u.ac.jp).

T. Ogitsu is with High Energy Accelerator Research Organization, Tsukuba 305-0801, Japan (email: ogitsu@ post.kek.jp).

M. Yoshimoto is with Japan Atomic Energy Agency, Tokai 319-1184, Japan (email: yoshimoto.masahiro@jaea.go.jp).

I. Watanabe and T. Yoshiyuki are with Toshiba Corporation, Tokyo 105-8001, Japan (email: ikuo4.watanabe@ toshiba.co.jp).
$B(r)=B_{0}\left(r / R_{0}\right)^{k}$

where $B_{0}$ is magnetic field at the magnet center, $r$ is distance from the accelerator center, $R_{0}$ is distance between the magnet center and the accelerator center, and $k$ is referred to as $k$-value [5]-[7]. The magnitude and the gradient of the magnetic field contribute to bending and focusing the beam, respectively.

We made a trial design of the FFAG accelerator for carbon therapy applying linear approximation [6]. The beam orbit and an outline of the accelerator design are shown in Fig. 1 and Table I, respectively. The type of lattice was FDF triplet radial sector. The radial sector FFAG accelerator needs the boundary of the sector to correspond to the radial direction of the beam ring, and uses two types of magnets whose polarities are opposite: a focus magnet and defocus magnet, which are hereinafter referred to as F magnet and D magnet, respectively. Alternating these magnets, the accelerator satisfies alternating gradient [5]-[7]. The packing factor is the ratio of total opening angle of magnets to 360 degrees. The F/D ratio is the ratio of bending angle of $\mathrm{F}$ magnets to that of $\mathrm{D}$ magnets. Although the parameters and the beam orbit of the accelerator were not optimized, magnets were designed considering this design.

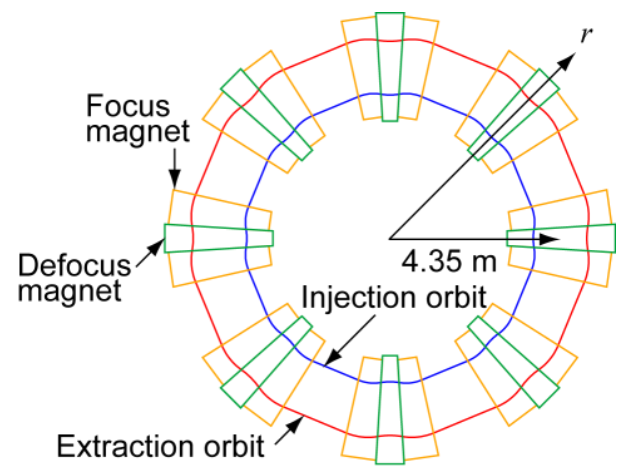

Fig. 1. Beam orbit of designed radial sector FFAG accelerator.

TABLE I Outline of Accelerator Design

\begin{tabular}{ccc}
\hline \hline Lattice, Packing factor, F/D ratio & FDF triplet, 0.56, 3.3 \\
$k$ value, Number of cells & 4,8 \\
Accelerated particle (Energy) & $\mathrm{C}^{6+}(20 \mathrm{MeV} / \mathrm{u}-400 \mathrm{MeV} / \mathrm{u})$ \\
Orbit radius & $3.6 \mathrm{~m}-5.1 \mathrm{~m}$ \\
\hline Type of magnet & F magnet & $\mathrm{D}$ magnet \\
\hline Integrated field at $r=4.35 \mathrm{~m}$ per magnet & $1.64 \mathrm{~T} \cdot \mathrm{m}$ & $1.06 \mathrm{~T} \cdot \mathrm{m}$ \\
Opening angle per magnet & 9.0 degrees & 7.2 degrees \\
\hline \hline
\end{tabular}




\section{THEORY FOR THREE-DIMENSIONAL DESIGN}

When designing the coil end of accelerator magnets wound with coated conductors, flat-wise bending, edge-wise bending and torsion, should be considered. In this paper, we applied differential geometry [8], [9], by which we can consider local bending curvature of conductors. Conductors are bent along the base curve, which is a curved line on the surface of a mandrel. One edge line of the tape is attached to the base curve. We show a conceptual view of three-dimensional winding in Fig. 2 (a), where three unit vectors $\boldsymbol{T}, \boldsymbol{n}$, and $\boldsymbol{b}$ are shown. These vectors are orthogonal and described in generalized Frenet-Serret equations shown as [8], [9]

$$
\frac{\mathrm{d} \boldsymbol{T}}{\mathrm{d} s}=\kappa_{\mathrm{n}} \boldsymbol{n}-\kappa_{\mathrm{g}} \boldsymbol{b}, \frac{\mathrm{d} \boldsymbol{n}}{\mathrm{d} s}=-\kappa_{\mathrm{n}} \boldsymbol{T}+\boldsymbol{t} \boldsymbol{b}, \frac{\mathrm{d} \boldsymbol{b}}{\mathrm{d} s}=\kappa_{\mathrm{g}} \boldsymbol{T}-\tau \boldsymbol{n},
$$

where $\boldsymbol{T}$ is the vector that corresponds to the tangent of the base curve, $\boldsymbol{n}$ is the vector that is perpendicular to the tape surface, and $\boldsymbol{b}$ is the vector that is perpendicular to $\boldsymbol{T}$ and $\boldsymbol{n} . \boldsymbol{s}$ is the distance along the base curve from the starting point of bending. $\kappa_{\mathrm{n}}\left(=\mathrm{d} \theta_{b} / \mathrm{d} s\right)$ is flat-wise bending curvature, $\kappa_{\mathrm{g}}\left(=\mathrm{d} \theta_{n} / \mathrm{d} s\right)$ is edge-wise bending curvature, and $\tau\left(=\mathrm{d} \theta_{T} / \mathrm{d} s\right)$ is torsion curvature [8], [9]. The generator vector $\boldsymbol{d}$ defines rulings, which are straight lines that generate a developable surface of the tape, and can be expressed with [8], [9]

$\boldsymbol{d}=-\left(\tau \boldsymbol{T}+\boldsymbol{\kappa}_{\mathrm{n}} \boldsymbol{b}\right)$

For the application of differential geometry to windings with coated conductors, we expanded the expression of the curvature parameters. When torsion is applied to tape without flat-wise bending $\left(\tau \neq 0, \kappa_{\mathrm{n}}=0\right), \boldsymbol{d}$ is parallel to the tangent of the base curve, which indicates that the tape surface is not developable. However, when flat-wise bending and torsion are combined $\left(\tau \neq 0, \quad \kappa_{\mathrm{n}} \neq 0\right)$, the tape surface is developable. Therefore, combination of flat-wise bending and torsion can be considered as generalized flat-wise bending, which is bending of developable surface. Conceptual view of developed surface of tape is shown in Fig. 2 (b). $w$ is the width of the tape. The distance $A$ between the base curve and the intersection of infinitely closely spaced two rulings, which are defined by $\boldsymbol{d}(s)$ and $\boldsymbol{d}(s+\Delta s)$, can be expressed with (4). When $0 \leq A \leq w$, two rulings intersect on the tape surface, which indicates that it is impossible to bend the tape. When $A<0$ or $w<A$, rulings do not intersect on the tape surface. $\sqrt{\tau^{2}+\kappa_{\mathrm{n}}^{2}}$ is bending angle between the two rulings. Therefore, generalized flat-wise bending curvature $\kappa_{\mathrm{f}}$ can be expressed with (5). $\alpha$ and $\beta$ are shown in Fig. 2 (b), and expressed with (6). $\delta$ is distance between base curve and a point on a ruling $(0 \leq \delta \leq w)$.

$$
\begin{aligned}
& A=\frac{\kappa_{\mathrm{n}}^{2}}{\kappa_{\mathrm{n}}(\mathrm{d} \tau / \mathrm{d} s)-\tau\left(\mathrm{d} \kappa_{\mathrm{n}} / \mathrm{d} s\right)} \\
& \kappa_{\mathrm{f}}=\lim _{\Delta s \rightarrow 0} \frac{\sqrt{\tau^{2}+\kappa_{\mathrm{n}}^{2}} \Delta s}{\alpha} \frac{A+\beta}{A-\delta}=\frac{\tau^{2}+\kappa_{\mathrm{n}}^{2}}{\kappa_{\mathrm{n}}} \frac{A}{A-\delta} \\
& \alpha=\left(\kappa_{\mathrm{n}} / \sqrt{\tau^{2}+\kappa_{\mathrm{n}}^{2}}\right) \Delta s, \beta=\left\{\tau \kappa_{\mathrm{n}} /\left(\tau^{2}+\kappa_{\mathrm{n}}^{2}\right)\right\} \Delta s
\end{aligned}
$$
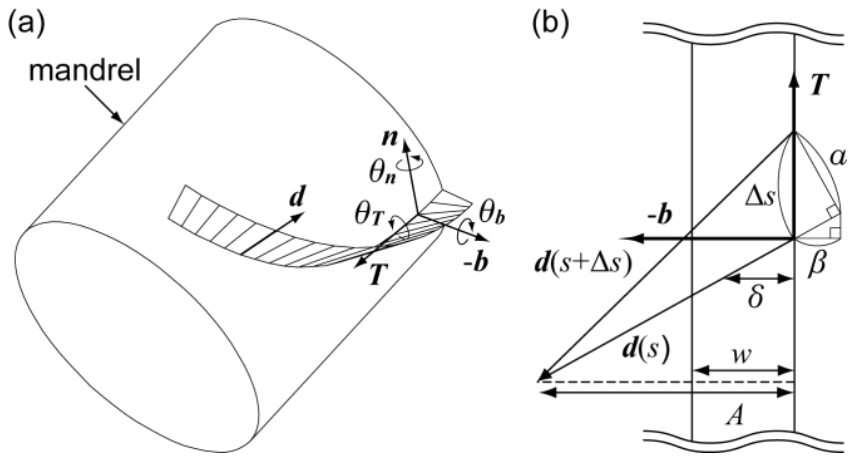

Fig. 2. (a) Conceptual view of three-dimensional winding. (b) Conceptual view of developed surface of tape.

When forming the coil-end, first, we determine the base curve, and assume that $\kappa_{\mathrm{g}}$ is zero. Therefore, $\boldsymbol{T}, \boldsymbol{n}, \boldsymbol{b}, \kappa_{\mathrm{n}}$, and $\tau$ can be calculated using (2). Second, using additional torsion $\theta_{T}{ }^{*}$, a new triad of vectors, $\boldsymbol{T}^{*}, \boldsymbol{n}^{*}$, and $\boldsymbol{b}^{*}$, and a new triad of curvatures, $\kappa_{\mathrm{n}}{ }^{*}, \kappa_{\mathrm{g}}{ }^{*}$, and $\tau^{*}$, can be calculated using [8], [9]

$$
\begin{aligned}
& \boldsymbol{T}^{*}=\boldsymbol{T}, \\
& \boldsymbol{n}^{*}=\cos \theta_{\boldsymbol{T}}^{*} \boldsymbol{n}+\sin \theta_{\boldsymbol{T}}^{*} \boldsymbol{b}, \boldsymbol{b}^{*}=\cos \theta_{\boldsymbol{T}}^{*} \boldsymbol{b}-\sin \theta_{\boldsymbol{T}}^{*} \boldsymbol{n}, \\
& \tau^{*}=\tau+\mathrm{d} \theta_{\boldsymbol{T}}^{*} / \mathrm{d} s, \\
& \kappa_{\mathrm{g}}^{*}=\sin \theta_{T}^{*} \kappa_{\mathrm{n}}, \kappa_{\mathrm{n}}^{*}=\cos \theta_{T}^{*} \kappa_{\mathrm{n}} .
\end{aligned}
$$

When determining $\theta_{T}{ }^{*}$ at both ends of the base curve, the tangent plane of the tape surface should be successive. $\theta_{T}{ }^{*}$ between both ends of the base curve is determined using the cubic spline function [9].

\section{Cross-Sectional Magnet Designs}

We designed the cross-section of the F magnets for the radial sector FFAG accelerator designed in section II. The magnets should generate the magnetic fields shown in (1), which can be expressed with the combination of multi-pole magnetic field components as [7]

$$
\begin{aligned}
& B(r)=B_{0}\left(r / R_{0}\right)^{k}=B_{0}\left\{\left(R_{0}+x\right) / R_{0}\right\}^{k} \\
& =B_{0}\left\{1+r_{0} \frac{k}{R_{0}} \frac{x}{r_{0}}+r_{0}^{2} \frac{k(k-1)}{2 ! R_{0}^{2}}\left(\frac{x}{r_{0}}\right)^{2}+\cdots\right\},
\end{aligned}
$$

where $r_{0}$ is reference radius, and $x$ is displacement from the magnet center on the $r$-axis. Surface current on the mandrel of magnet that generates each multi-pole magnetic field component can be calculated. Using the combination of these surface currents, we optimized the positions of conductors and minimized the multi-pole field error. The effect of the iron yoke was considered by the image-current method [10].

We assumed magnets wound with a bundle of ten 5-mm-wide and 0.2-mm-thick coated conductors. The designed magnets consist of pairs of layers. As shown in the inset of Fig. 3 (a), one layer consists of bundled tapes stacked face-to-back along the circumference of cross-section of the mandrel. Space between two adjacent bundles is $0.1 \mathrm{~mm}$, and there is no space between two adjacent conductors in one bundle. Space between two adjacent pairs of layers is $5 \mathrm{~mm}$, and space between pair of 
The distribution and the histogram of the magnetic fields at layers is $0.5 \mathrm{~mm}$. For simplicity, magnetization current was neglected. Vertical aperture of mandrel should be compressed to reduce the leakage fields. Therefore, we designed two types of magnets; a circle magnet whose mandrel is circle-shaped and an ellipse magnet whose mandrel is ellipse-shaped. We allowed operation of the magnet up to 500 A per tape.

Outlines and cross-section of the designed $\mathrm{F}$ magnets are shown in Table II and Fig. 3, respectively. The radius of the mandrel and the inner radius of the iron yoke of the circle magnet were $1.2 \mathrm{~m}$ and $1.4 \mathrm{~m}$, respectively. The semi-major axis and the semi-minor axis of the mandrel and the inner radius of the iron yoke of the ellipse magnet were $1.4 \mathrm{~m}, 0.8 \mathrm{~m}$ and $1.5 \mathrm{~m}$, respectively. Both magnets had 10 layers.

The field quality of the designed magnets was considered using local $k$ value expressed with [7]

local $k=\frac{\mathrm{d} B}{\mathrm{~d} r} \frac{r}{B(r)}$.

The distributions of local $k$ of the designed $\mathrm{F}$ magnets are shown in Fig. 4 (left axis). The target value was 4, and errors were successfully reduced at the good field region $\pm 0.77 \mathrm{~m}$.

TABLE II Outlines of Cross-Sectional Focus Magnet Designs

\begin{tabular}{ccc}
\hline \hline Shape of mandrel & Circle & Ellipse \\
\hline Radius of mandrel & $1.2 \mathrm{~m}$ & $1.4 \mathrm{~m} / 0.8 \mathrm{~m}$ \\
Inner radius of iron yoke & $1.4 \mathrm{~m}$ & $1.5 \mathrm{~m}$ \\
Distance between magnet center and & $4.35 \mathrm{~m}$ & $4.35 \mathrm{~m}$ \\
accelerator center & $\pm 0.77 \mathrm{~m}$ & $\pm 0.77 \mathrm{~m}$ \\
Good field region & $414 \mathrm{~A}$ & $415 \mathrm{~A}$ \\
Operation current per tape & $2.41 \mathrm{~T}$ & $2.41 \mathrm{~T}$ \\
Magnetic field at magnet center & $9.27 \mathrm{~T}$ & $9.15 \mathrm{~T}$ \\
Peak field & $1910 \times 10$ & $1890 \times 10$ \\
Number of turns & 10 & 10 \\
Number of layers &
\end{tabular}

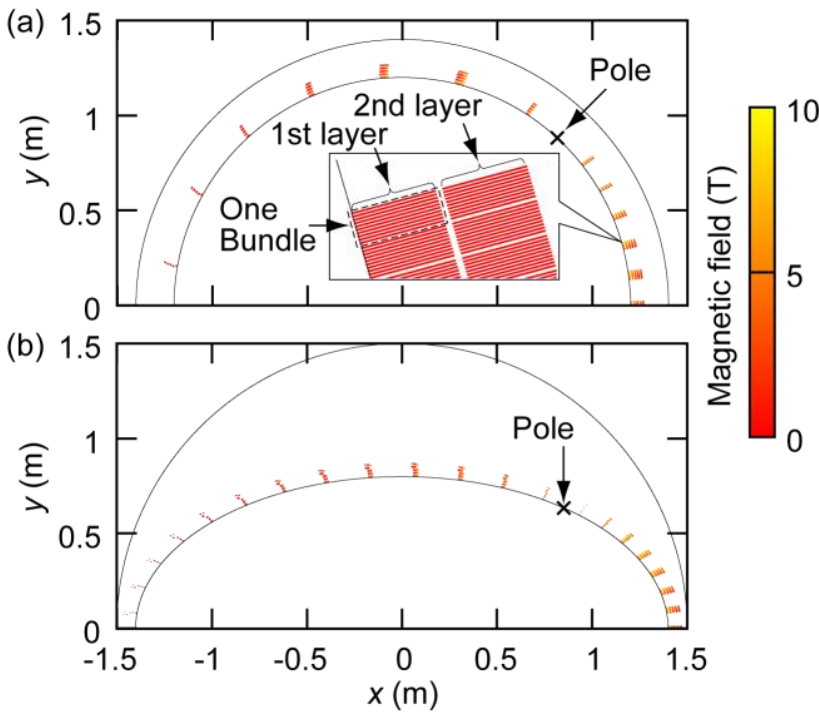

Fig. 3. Cross-section of designed F magnets. (a) Circle-shaped mandrel. (b) Ellipse-shaped mandrel. (Color shows magnitude of magnetic fields at conductors.) conductors of the designed F magnets are shown in Fig. 3 and Fig. 5, respectively. The peak fields of the circle magnet and the ellipse magnet were $9.27 \mathrm{~T}$ and $9.15 \mathrm{~T}$, respectively. The maximum magnetic field at the surface of iron yoke was about 4-5 T.

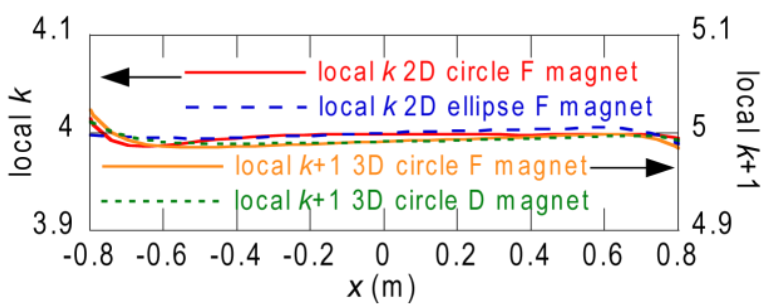

Fig. 4. Local $k$ of designed $\mathrm{F}$ magnets (left axis). Local $k+1$ of designed magnets (right axis).

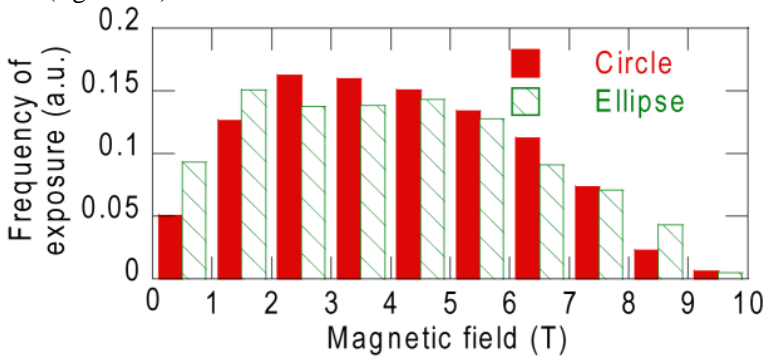

Fig. 5. Histogram of magnetic field at conductors of designed $\mathrm{F}$ magnets.

\section{ThreE-Dimensional Magnet Designs}

We designed three-dimensional shapes of magnets for the FFAG accelerator designed in section II. When designing the coil-end and calculating the bending curvature of conductors, we applied the differential geometry shown in section III. The tangent of the base curve should be successive and should be parallel to the straight section of the magnet at the starting point of bending. When determining the base curve, first, we defined the radial section, in which base curve traced to the beam orbit plane should be straight and parallel to the boundary of a magnet sector. Because it was hard to connect the radial section and the straight section directly, we defined a joint section that connects them. In the joint section, the base curve was ellipse-shaped on the developed plane of the mandrel.

In the radial section, additional torsion $\theta_{T}^{*}$, which is explained in section III, was fixed to -90 degrees. Therefore, conductors were bent edge-wise only, which indicates that one edge line of the tape is on the mandrel, and the wide face of tape is almost perpendicular to the mandrel. In the case of usual cosine-theta magnets consisting of NbTi Rutherford cables, three-dimensional-shaped spacers are needed at the coil-end. When designing magnets wound with single coated conductors, much more spacers would be needed. Whereas, since we allowed edge-wise bending, the tape faces were almost perpendicular to the mandrel and parallel to adjacent tape faces in the radial section. Therefore, no spacer is needed between adjacent conductors, and number of spacers is reduced.

Since the positions of the tape face in the radial section and the straight section have been defined, we determined $\theta_{T}{ }^{*}$ at both ends of the base curve in the joint section so as to make the tangent plane of the tape face to be successive. Generalized flat-wise bending curvature was calculated using (5). Bending 
radius equals the reciprocal number of curvature. Edge-wise bending strain was calculated using

$$
\varepsilon_{\mathrm{g}}=\{(w / 2)-\delta\} /\left\{\left(1 / \kappa_{\mathrm{g}}\right)-(w / 2)\right\}
$$

where $\delta$ is distance between base curve and a point on a tape face $(0 \leq \delta \leq w)$. We allowed the generalized flat-wise bending radius to be as little as $20 \mathrm{~mm}$, and the edge-wise bending strain to be as high as $0.3 \%$ [11], [12].

To consider the leakage field of the magnet, we integrated the generated magnetic field along the arc on the beam orbit plane. The center of the arc corresponds to the accelerator center. The central angle of the arc was 45 degrees. The integrated field $B_{L}$ should satisfy [7]

$$
B_{L}(r)=B_{L 0}\left(r / R_{0}\right)^{k+1} .
$$

We optimized the positions of conductors to minimize the error of $B_{L}$ iterating these two designs: the cross-sectional design, which reduces the error of $B_{L}$, and the coil-end design. Generated magnetic fields were calculated using Biot-Savart law, and the effect of the iron yoke was considered by the image-current method.

We assumed magnets wound in bundles and layers in the same way as detailed in section IV. For simplicity, magnetization current was neglected, and we assumed a circle-shaped mandrel. We allowed operation of the magnet up to 500 A per tape.

Outlines of the designed magnets and a bird's-eye view of the designed F magnet are shown in Table III and Fig. 6 (a), respectively. The radius of the mandrel and the inner radius of the iron yoke of the $\mathrm{F}$ magnet were $1.2 \mathrm{~m}$ and $1.4 \mathrm{~m}$, respectively. The radius of the mandrel and the inner radius of the iron yoke of the $\mathrm{D}$ magnet were $1.3 \mathrm{~m}$ and $1.5 \mathrm{~m}$, respectively. Both magnets had 10 layers. Fig. 6 (b) shows top view of inner-most layer of the designed $F$ magnet. The minimum generalized flat-wise bending radius was $50.3 \mathrm{~mm}$, and the maximum edge-wise bending strain was $0.275 \%$.

The field quality of the designed magnets was considered using local $k+1$ value expressed with [7]

TABLE III Outlines of Three-Dimensional Magnet Designs

\begin{tabular}{ccc}
\hline \hline Type of magnet & F magnet & D magnet \\
\hline Radius of mandrel & $1.2 \mathrm{~m}$ & $1.3 \mathrm{~m}$ \\
Inner radius of iron yoke & $1.4 \mathrm{~m}$ & $1.5 \mathrm{~m}$ \\
Distance between magnet center and \\
accelerator center & $4.35 \mathrm{~m}$ & $4.35 \mathrm{~m}$ \\
Good field region & $\pm 0.77 \mathrm{~m}$ & $\pm 0.77 \mathrm{~m}$ \\
Operation current per tape & $461 \mathrm{~A}$ & $465 \mathrm{~A}$ \\
Magnetic field at magnet center & $1.09 \mathrm{~T}$ & $0.676 \mathrm{~T}$ \\
Integrated field at $r=4.35$ m per magnet & $1.64 \mathrm{~T} \cdot \mathrm{m}$ & $1.06 \mathrm{~T} \cdot \mathrm{m}$ \\
Number of turns & $2158 \times 10$ & $2066 \times 10$ \\
Number of layers & 10 & 10 \\
Length of conductors per magnet & $87.2 \mathrm{~km}$ & $79.0 \mathrm{~km}$ \\
\hline \hline
\end{tabular}

local $k+1=\frac{\mathrm{d} B_{L}}{\mathrm{~d} r} \frac{r}{B_{L}(r)}$

The distributions of local $k+1$ of the designed magnets are shown in Fig. 4 (right axis). The target value of local $k+1$ was 5, and errors were successfully reduced at the good field region $\pm 0.77 \mathrm{~m}$.

(a)

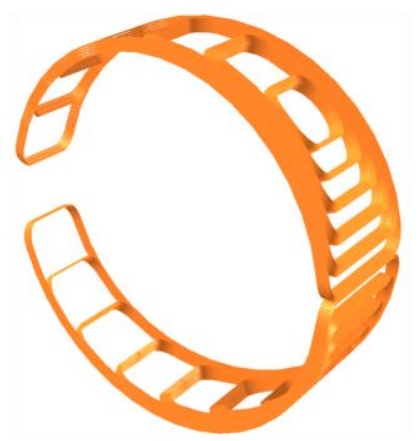

(b)

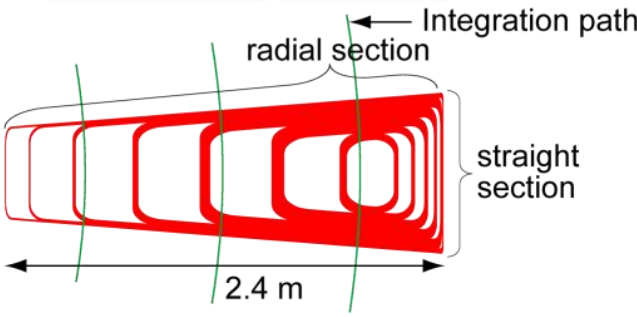

Fig. 6. (a)Bird's-eye view of designed $\mathrm{F}$ magnet. (b)Top view of inner-most layer of designed $\mathrm{F}$ magnet.

\section{SUMMARY}

We proposed a theory for three-dimensional winding design with coated conductors, which is applicable to various types of accelerator magnets with three-dimensional shape. Using this theory, we successfully designed coil-dominated magnets for a radial sector FFAG accelerator for carbon therapy. No spacer is needed between adjacent conductors at the coil-end of the designed magnets, and it is easy to apply bundled conductors.

For simplicity, the circle-shaped mandrel was assumed in three-dimensional magnet designs. However, the vertical aperture of the mandrel should be compressed to reduce the leakage fields of the magnet, as shown in the cross-sectional magnet designs. A next step is designing magnets for a spiral sector FFAG accelerator [5], so as to reduce the quantity of conductors and magnetic fields.

\section{REFERENCES}

[1] R. Gupta et al., "High field HTS R\&D solenoid for muon collider," IEEE Trans. Appl. Supercond., vol. 21, no. 3, pp. 1884-1887, June, 2011.

[2] K. Hatanaka et al., "Development of a HTS magnet and application to a beam scanner," Nucl. Instr. Meth. in Phys. Res. Section A, vol. 571, no. 3, pp. 583-587, Feb., 2007.

[3] K. Takahashi et al., "Magnetic field design of dipole magnet wound with coated conductor considering its current transport characteristics," IEEE Trans. Appl. Supercond., vol. 21, no. 3, pp. 1833-1837, June, 2011.

[4] S. Russenschuck, Field computation for accelerator magnets. Weinheim: WILEY-VCH, 2010, pp. 293-326.

[5] K. R. Symon, D. W. Kerst, L. W. Jones, L. J. Laslett, and K. M. Terwilliger, "Fixed-field alternating-gradient particle accelerators," Phys. Rev., vol. 103, no. 6, pp. 1837-1859, June, 1956.

[6] S. Machida, Y. Mori, and R. Ueno, "Beam optics design of an FFAG synchrotron," Proceedings of European Particle Accelerator Conference, pp. 557-559, 2000. 
[7] T. Obana et al., "Magnetic field design of superconducting magnet for a FFAG accelerator," IEEE Trans. Appl. Supercond., vol. 15, no. 2, pp. 1185-1188, June, 2005.

[8] B. Auchmann, and S. Russenschuck, "Coil end design for superconducting magnets applying differential geometry methods," IEEE Trans. Magn., vol. 40, no. 2, pp. 1208-1211, Mar.., 2004.

[9] S. Russenschuck, Field computation for accelerator magnets. Weinheim: WILEY-VCH, 2010, pp. 609-636.
[10] S. Russenschuck, Field computation for accelerator magnets. Weinheim: WILEY-VCH, 2010, pp. 209-216.

[11] D. C. van der Laan, and J. W. Ekin, "Dependence of the critical current of $\mathrm{YBa}_{2} \mathrm{Cu}_{3} \mathrm{O}_{7-\delta}$ coated conductors on in-plane bending," Supercond. Sci. Technol., vol. 21, no. 115002, Sept., 2008.

[12] M. Sugano, K. Shikimachi, N. Hirano, and S. Nagaya, "Simultaneously bending and tensile strain effect on critical current in YBCO coated conductors," Physica C, vol. 463-465, pp. 742-746, June, 2007. 